\title{
Accurate and Efficient BER Evaluation for High-Speed OFDM Systems Impaired by TI-ADC Circuit's Gain Mismatch
}

\author{
Vo-Trung-Dung Huynh, Nele Noels, Heidi Steendam \\ Department of Telecommunications and Information Processing, Ghent University \\ \{votrungdung.huynh, nele.noels, heidi.steendam\}@ugent.be
}

\begin{abstract}
This paper presents an efficient procedure to numerically evaluate the exact bit error rate of a rectangular quadrature amplitude modulated-orthogonal frequency division multiplexing (OFDM) system that is impaired by the gain mismatch of a time-interleaved analog-to-digital converter. As opposed to previous contributions, no approximations are involved in this procedure. The obtained results allow to accurately analyze the effect of this type of mismatch on the performance of practical high-speed OFDM systems. The accuracy and efficiency of the proposed technique are demonstrated by comparing analytical results with brute-force Monte-Carlo simulations.
\end{abstract}

Keywords-Bit error rate, OFDM, time-interleaved analog-todigital converter, gain mismatch, Monte-Carlo simulation.

\section{INTRODUCTION}

Thanks to its high spectral efficiency and tolerance against channel dispersion, orthogonal frequency division multiplexing (OFDM) has recently been introduced to many multi-Gigabit broadband wired and wireless communication systems [1]. Such high-speed OFDM systems require a high sampling rate analog-to-digital converter (ADC) placed prior to the baseband digital signal processing unit at the receiver. However, the operating sampling rate of a regular ADC is restricted by the physical constrains of the employed technology [2]. A timeinterleaved (TI) structure of $L$ identical parallel ADCs allows to achieve such high sampling rates. The $L$ sub-ADCs each sample the analog input signal at a rate $\frac{1}{L T_{s}}$ with $\frac{1}{T_{s}}$ the overall sampling rate, where the sampling instants of the different subADCs are equidistantly spaced in time with interspacing $T_{s}$.

A bottleneck of the use of TI-ADCs is the mismatch between the sub-ADCs, which can cause a significant system performance degradation [3-6]. In particular, the effect of gain mismatch on OFDM bit error rate (BER) performance was studied in $[5,6]$. In [5], the authors evaluated the effect of TI-ADC's gain mismatch on the OFDM-BER performance in an AWGN channel by means of a tedious brute-force MonteCarlo (BF-MC) simulation. In [6], an approximate closed-form BER expression was derived, again for an AWGN channel by adopting a Gaussian approximation (GA) for the inter-carrier interference (ICI) term caused by gain mismatch. However, this approach was seen to yield inaccurate results when the number $L$ of sub-ADCs is low. In order to overcome the limitations of the approaches from $[5,6]$, we propose an efficient semi-analytic (SA) approach to evaluate BER values for OFDM systems affected by TI-ADC's gain mismatch. The approach consists in the analytical computation of the
BER conditioned on the data sequence $\mathbf{X}$ and the channel vector $\mathbf{H}$, i.e., $B E R_{\mid \mathbf{X}, \mathbf{H}}$, followed by a numerical averaging of $B E R_{\mid \mathbf{X}, \mathbf{H}}$ over the joint distribution of $\mathbf{X}$ and $\mathbf{H}$ using MC technique. For AWGN and static channels, standard MC can be applied. For Rayleigh fading channels, an appropriate importance sampling (IS) technique is proposed to reduce the time required to perform the MC simulation.

The paper is organized as follows. Section II describes the system model. An exact semi-analytic BER evaluation procedure is derived in Section III. Section IV provides numerical results to validate the accuracy and efficiency of the proposed procedure. Our conclusion is given in Section V.

\section{SYSTEM DESCRIPTION}

Fig. 1 illustrates the block diagram of the considered OFDM system. The discrete baseband OFDM signal transmitted in one OFDM symbol period is given by:

$$
s_{k}=\frac{1}{\sqrt{N}} \sum_{a=0}^{N-1} X_{a} e^{j 2 \pi \frac{a k}{N}}, \quad 0 \leq k \leq N-1,
$$

where $N$ is the number of sub-carriers in an OFDM system, and $\mathbf{X}=\left(X_{0}, X_{1}, \ldots, X_{N-1}\right)^{T}$ is a vector consisting of data symbols taken from a unit-energy rectangular $M_{I} \times M_{Q^{-}}$ QAM constellation, in which $\left(\log _{2} M_{I}+\log _{2} M_{Q}\right)$ transmitted bits, i.e., $b_{u}^{\left(X_{n}^{I}\right)}$ with $u=1,2, \ldots, \log _{2} M_{I}$ and $b_{u}^{\left(X_{n}^{Q}\right)}$ with $u=1,2, \ldots, \log _{2} M_{Q}$, are mapped on the constellation symbol $X_{n}$. To eliminate inter-symbol interference (ISI), a cyclic prefix (CP), whose length is larger than the maximum delay spread of the channel, is applied. Before transmission, the OFDM signal passes through a digital-to-analog converter (DAC) and a transmit filter. At the receiver, assuming timing synchronization and matched filtering, the received waveform after CP removal is sampled by a TI-ADC with $L$ parallel sub-ADCs. The TI-ADC is assumed to have a sufficiently high resolution so that the quantization noise can be neglected [7]. Furthermore, since in practice, the gain values of the subADCs in a TI-ADC are only slowly time varying [8], we model them as constants over the duration of an OFDM symbol. The output of the TI-ADC with gain mismatch can be expressed as [6]:

$$
r_{k}=\sum_{l=0}^{L-1} \sum_{q=-\infty}^{+\infty}\left(1+d g_{l}\right)\left(\sqrt{E_{s}} \cdot s_{k} \otimes h_{k}+w_{k}\right) \cdot \delta_{k-q L-l},
$$




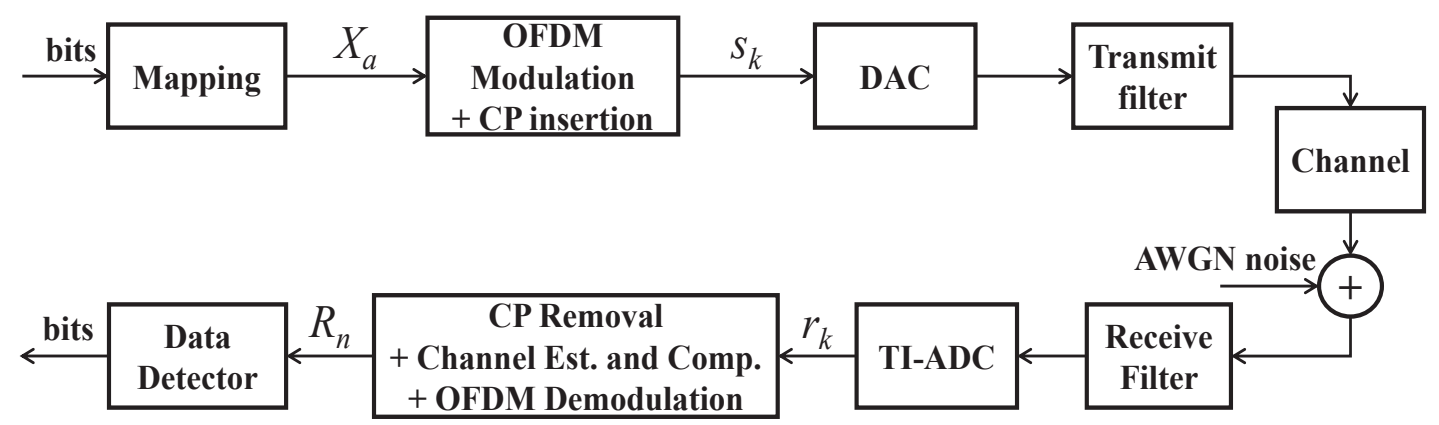

Fig. 1. Block diagram of an OFDM system with a TI-ADC at the receiver.

where $r_{k}$ denotes the $k$-th received sample, $h_{k}$ is the sampled impulse response of the channel, $\otimes$ denotes the discrete convolution operation, $s_{k}$ is defined by (1), $d g_{l}$ is the gain mismatch value of the $l$-th sub-ADC expressed relative to the transmitted symbol energy $E_{s}, \delta$ denotes the discrete dirac function, and $w_{k}$ are independently and identically distributed (i.i.d.) AWGN noise samples with zero mean and variance $\frac{N_{0}}{2}$ per dimension. Further, in (2), we assume a slowly varying channel, so that the channel impulse response remains roughly constant over a symbol period [9]. Before data detection, the receiver employs a zero-forcing equalizer to compensate the effect of the channel. The output of the equalizer is given by:

$$
\begin{aligned}
& R_{n}=\frac{1}{H_{n} \sqrt{N}} \sum_{k=0}^{N-1} r_{k} e^{-j 2 \pi \frac{k n}{N}} \\
&=\sqrt{E_{s}}\left(1+D G_{0}\right) X_{n}+\sqrt{E_{s} \frac{A_{n}}{H_{n}}+\frac{\Phi_{n}}{H_{n}},} \\
& 0 \leq n \leq N-1,
\end{aligned}
$$

where $H_{n}$ is the frequency channel response and $D G_{i}$ is given by:

$$
D G_{i}=\frac{1}{L} \sum_{l=0}^{L-1} d g_{l} e^{-j 2 \pi \frac{i l}{L}}
$$

Further, in (3), the interference contribution $A_{n}$ is defined as:

$$
A_{n}=\sum_{i=1}^{L-1} D G_{i} X_{t} H_{t}
$$

with $t=\bmod (n-i N / L, N)$ denoting the remainder after division of $n-i N / L$ by $N$, and the noise contribution $\Phi_{n}$ is written as:

$$
\Phi_{n}=\sum_{i=0}^{L-1} D G_{i} W_{t}+W_{n},
$$

with $W_{n}$ the i.i.d. AWGN noise samples in frequency domain, In (3), it is assumed that the number $L$ of sub-ADCs is a power of 2 , and the ratio $\frac{N}{L}$ between the DFT size and the number of sub-ADCs is an integer value. Extension to non-integer values of $\frac{N}{L}$ is straightforward ${ }^{1}$. The quantities $R_{n}$ (3) are used to detect the bits corresponding to $X_{n}$ by mapping $R_{n}$ to the nearest constellation point and applying the inverse mapping

\footnotetext{
${ }^{1}$ In that case, $R_{n}$ (3) becomes $R_{n}=\sqrt{E_{s}} X_{n}+$ $\sqrt{E_{s}} \hat{A}_{n} / H_{n}+\hat{\Phi}_{n} / H_{n}$, where the interference contribution is $\hat{A}_{n}=\sqrt{E_{s}} \sum_{i=0}^{L-1} D G_{i} \sum_{a=0}^{N-1} X_{a} H_{a} \operatorname{sinc}(a-t)$ and the noise contribution $\hat{\Phi}_{n}=\sum_{i=0}^{L-1} D G_{i} \sum_{a=0}^{N-1} W_{a} \operatorname{sinc}(a-t)+W_{n}$, with $\operatorname{sinc}(x)=\frac{\sin (\pi x)}{\pi x}$.
}

rule; the resulting estimated bits are donated $b_{u}^{\left(R_{n}^{I}\right)}$ with $u=$ $1,2, \ldots, \log _{2} M_{I}$ and $b_{u}^{\left(R_{n}^{Q}\right)}$ with $u=1,2, \ldots, \log _{2} M_{Q}$.

\section{BER ANALYSIS}

In this section, first an exact BER expression is derived for a given data sequence $\mathbf{X}$ and a given frequency channel response vector $\mathbf{H}$. Assuming $N_{d}$ data-modulated subcarriers $^{2}$, the BER for given $\mathbf{X}$ and $\mathbf{H}$, i.e., $B E R_{\mid \mathbf{X}, \mathbf{H}}$, can be decomposed as:

$$
B E R_{\mid \mathbf{X}, \mathbf{H}}=\frac{1}{N_{d}} \sum_{n \in I_{d}} B E R_{\mid \mathbf{X}, \mathbf{H}, n},
$$

where $B E R_{\mid \mathbf{X}, \mathbf{H}, n}$ denotes the conditional $B E R_{\mid \mathbf{X}, \mathbf{H}}$ for the $n$-th data-modulated sub-carrier and $I_{d}$ is the set of indices of the modulated sub-carriers, i.e., $I_{d} \subset\{0,1, \ldots, N-1\}$. Further, as each sub-carrier carries a rectangular QAM symbol $X_{n}$, formed by a sequence of $\left(\log _{2} M_{I}+\log _{2} M_{Q}\right)$ independent and equiprobable bits using the binary reflected Gray code (BRGC) bit mapping rule [12], $B E R_{\mid \mathbf{X}, \mathbf{H}, n}$ in (7) can be further decomposed as:

$$
B E R_{\mid \mathbf{X}, \mathbf{H}, n}=\frac{1}{m_{I}+m_{Q}} \sum_{\beta, u} B E R_{\mid \mathbf{X}, \mathbf{H}, n, u}^{\beta},
$$

where $\beta \in\{I, Q\}$ refers to the in-phase (I) and quadrature (Q) dimension of the signal, $m_{\beta}=\log _{2} M_{\beta}, u \in\left\{1,2, \ldots, m_{\beta}\right\}$, and $B E R_{\mid \mathbf{X}, \mathbf{H}, n, u}^{\beta}$ is the BER corresponding to the bit $b_{u}^{\left(X_{n}^{\beta}\right)}$. Taking into account (3), $B E R_{\mid \mathbf{X}, \mathbf{H}, n, u}^{\beta}$ can be written as:

$$
\begin{aligned}
& \operatorname{BER}_{\mid \mathbf{X}, \mathbf{H}, n, u}^{\beta}= \\
& \operatorname{Pr}\left[\left(\left(1+D G_{0}\right) X_{n}+\frac{A_{n}}{H_{n}}+\frac{\Phi_{n}}{\sqrt{E_{s}} H_{n}}\right)^{\beta} \notin \Omega_{u, X_{n}} \mid \mathbf{X}, \mathbf{H}\right],
\end{aligned}
$$

where $(z)^{\beta}$ is defined as:

$$
(z)^{\beta}=\left\{\begin{array}{ll}
\Re\{z\} & , \text { if } \beta=I \\
\Im\{z\} & , \text { if } \beta=Q
\end{array},\right.
$$

with $\Re\{z\}$ and $\Im\{z\}$ denoting the real and imaginary part of $z$, respectively. Further, $\Omega_{u, X_{n}}$ in (9) is the interval of $\frac{1}{\sqrt{E_{s}}}\left(R_{n}\right)^{\beta}$, with $R_{n}$ from (3), for which the received bit $b_{u}^{\left(R_{n}^{\beta}\right)}$ equals the transmitted bit $b_{u}^{\left(X_{n}^{\beta}\right)}$. For the

\footnotetext{
${ }^{2}$ In practice, in many OFDM systems, not all $N$ sub-carriers are used for data transmission. For instance, a few sub-carriers near the edges (i.e., the guard band) are not modulated to achieve a sufficient transition band at the bandwidth boundaries [10].
} 


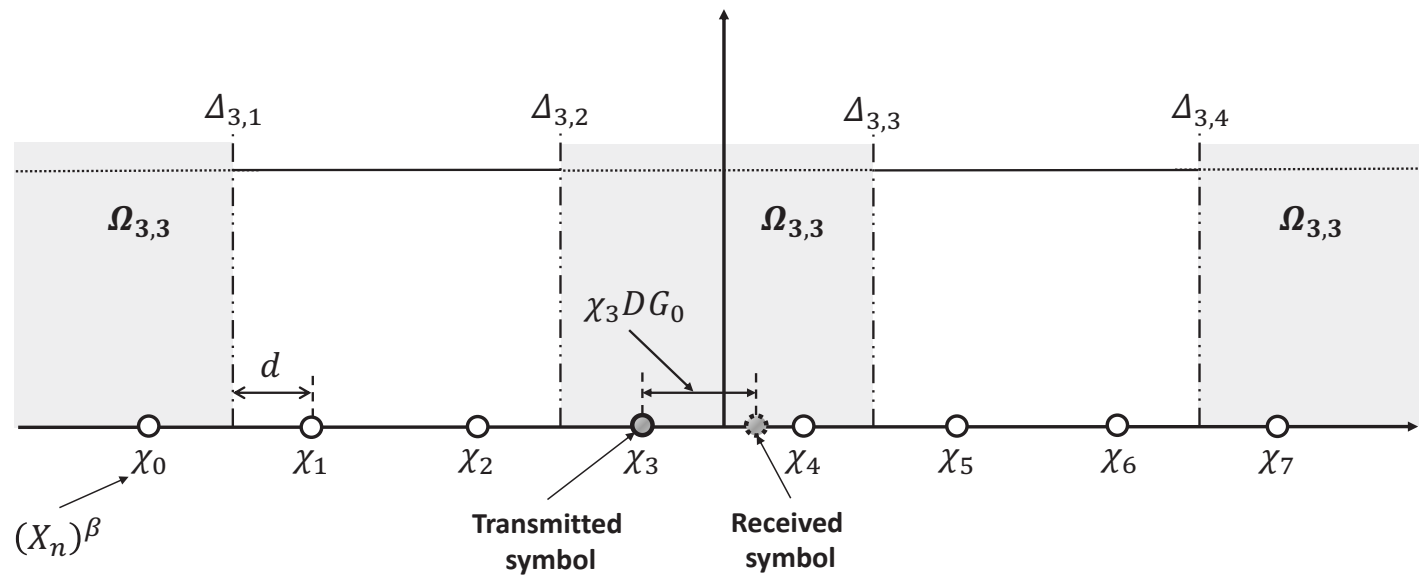

Fig. 2. An illustration of the decision regions and boundaries required to derive the $B E R_{\mid \mathbf{X}, \mathbf{H}, n, u}^{\beta}$ expression for $M_{\beta}=8, u=3, v=3$.

BRGC mapping, the boundaries of the interval $\Omega_{u, X_{n}}$ can be expressed as [13] (see an example in Fig. 2): $\Delta_{u, y}=$ $\left((2 y-1) \cdot 2^{m_{\beta}-u+1}-M_{\beta}\right) d_{\beta}$, where $d_{\beta}$ denotes the half minimum Euclidean distance in the $\beta$-dimension [11] and $y \in\left\{1,2, \ldots, 2^{u-1}\right\}$. Note that for a given data sequence and a given channel, $A_{n}$ (5) can be considered as constant. Further, the noise term $\Phi_{n}$ is an i.i.d. complex Gaussian random variable with zero mean and variance $\sigma^{2}=$ $\frac{N_{0}}{2}\left(1+2 D G_{0}+\sum_{i=0}^{L-1}\left|D G_{i}\right|^{2}\right)$ per dimension, where $|z|$ denotes the absolute value of $z$. Hence, it follows that $B E R_{\mid \mathbf{X}, \mathbf{H}, n, u}^{\beta}$ from (9) is of the following form:

$$
\begin{aligned}
& \operatorname{BER}_{\mid \mathbf{X}, \mathbf{H}, n, u}^{\beta} \\
& =\frac{1}{2} \sum_{y=1}^{F_{u, v}} \lambda_{u, v, y} \operatorname{erfc}\left(\Gamma_{u, v, y}\left(X_{n}, H_{n}\right)\right) \\
& +\frac{1}{2} \sum_{y=F_{u, v}+1}^{2^{u-1}} \rho_{u, v, y} \operatorname{erfc}\left(-\Gamma_{u, v, y}\left(X_{n}, H_{n}\right)\right) .
\end{aligned}
$$

In (11), the BER consists of two sums, corresponding to the decision boundaries (for given $u$ ) at the left and right side of $\left(X_{n}\right)^{\beta}$, respectively. The number of terms in the sums depend on $F_{u, v}$, which indicates the number of the boundaries left of the considered constellation symbol. For the BRGC mapping rule, we have: $F_{u, v}=\left\lfloor(2 v+1) 2^{-\left(m_{\beta}-u+2\right)}+2^{-1}\right\rfloor$, where $\lfloor z\rfloor$ denotes the largest integer smaller than $z$ and $v=$ $\frac{1}{2}\left(\left(X_{n}\right)^{\beta} / d_{\beta}+M_{\beta}-1\right)$. Further, $\operatorname{erfc}($.$) is the comple-$ mentary error function (erfc-function) defined by: $\operatorname{erfc}(x)=$ $\frac{2}{\sqrt{\pi}} \int_{x}^{\infty} e^{-z^{2}} d z$, and the argument $\Gamma_{u, v, y}\left(X_{n}, H_{n}\right)$ of the erfcfunction in (11) is given by:

$$
\begin{aligned}
& \Gamma_{u, v, y}\left(X_{n}, H_{n}\right)= \\
& \left(\left(1+D G_{0}\right)\left(X_{n}\right)^{\beta}+\left(\frac{A_{n}}{\left|H_{n}\right| e^{j \theta}{ }^{\beta}}\right)^{\beta}-\Delta_{u, y}\right)\left|H_{n}\right| \sqrt{\frac{E_{s}}{2 \sigma^{2}}},
\end{aligned}
$$

with $\theta_{n}=\arctan \left(\left(H_{n}\right)^{Q} /\left(H_{n}\right)^{I}\right)$. Finally, the pre-factors $\lambda_{u, v, y}=(-1)^{\left\lfloor 2^{u-2-m_{\beta}}\left(\left(\left(X_{n}\right)^{\beta}-\Delta_{u, y}\right) / d_{\beta}-1\right)\right\rfloor}$ and $\rho_{u, v, y}=$ $(-1)^{\left\lfloor 2^{u-2-m_{\beta}}\left(-\left(\left(X_{n}\right)^{\beta}-\Delta_{u, y}\right) / d_{\beta}-1\right)\right\rfloor}$ take the values +1 or
-1 . Substituting (8) and (11) into (7), we obtain

$$
\begin{aligned}
& B E R_{\mid \mathbf{X}, \mathbf{H}}=\frac{1}{2 N_{d}\left(m_{I}+m_{Q}\right)} . \\
& \sum_{n, \beta, u}\left(\sum_{y=1}^{F_{u, v}} \lambda_{u, v, y} \operatorname{erfc}\left(\Gamma_{u, v, y}\left(X_{n}, H_{n}\right)\right)\right. \\
& \left.\quad+\sum_{y=F_{u, v}+1}^{2^{u-1}} \rho_{u, v, y} \operatorname{erfc}\left(-\Gamma_{u, v, y}\left(X_{n}, H_{n}\right)\right)\right) .
\end{aligned}
$$

The BER expression for an $M_{s}$-ary square QAM constellation is obtained by setting in (13) $M_{I}=M_{Q}=\sqrt{M_{s}}$ and $d_{I}=d_{Q}$. Similarly, the BER expression for an $M_{p}$-ary PAM constellation is derived by setting $M_{I}=M_{p}, M_{Q}=1$ and the sum over the $\beta=Q$ dimension disappears.

We will now use (13) to evaluate the BER, by averaging $B E R_{\mid \mathbf{X}, \mathbf{H}}$ over the statistics of $\mathbf{X}$ and $\mathbf{H}$. However, due to the complex dependency of (13) on $\mathbf{X}$ and $\mathbf{H}$, a closed-form expression of the average BER cannot be obtained. Hence, we resort to MC simulation to obtain an unbiased estimate of the BER. A standard MC estimator computes the BER as [14]:

$$
B E R_{M C}=\frac{1}{N_{M C}} \sum_{j=1}^{N_{M C}} B E R_{\mid \mathbf{X}_{j}, \mathbf{H}_{j}},
$$

where $N_{M C}$ is the number of simulation runs in the MC experiment. Each run requires the re-evaluation of (13). The accuracy of (14) increases with the value of $N_{M C}$. In the case of AWGN or static channels (i.e., fibre-optic or wired RF links), the BER is efficiently evaluated by using (14). However, for Rayleigh channels, a very large $N_{M C}$ is typically required to obtain an accurate estimate for lower BER values. This is mainly due to the low occurrence probability of bad channels. To reduce the computation time, as an alternative, the unbiased self-normalized importance sampling (IS) estimator [14] can be employed. In that case, the average BER for Rayleigh channels is computed as:

$$
B E R_{I S}=\frac{1}{N_{I S}} \sum_{j=1}^{N_{I S}} B E R_{\mid \mathbf{X}_{j}, \mathbf{H}_{i}, \mathbf{w}_{j}},
$$

using $N_{I S}$ simulation runs in the IS experiment. In contrast to (14), where each channel evaluation is assigned the same 


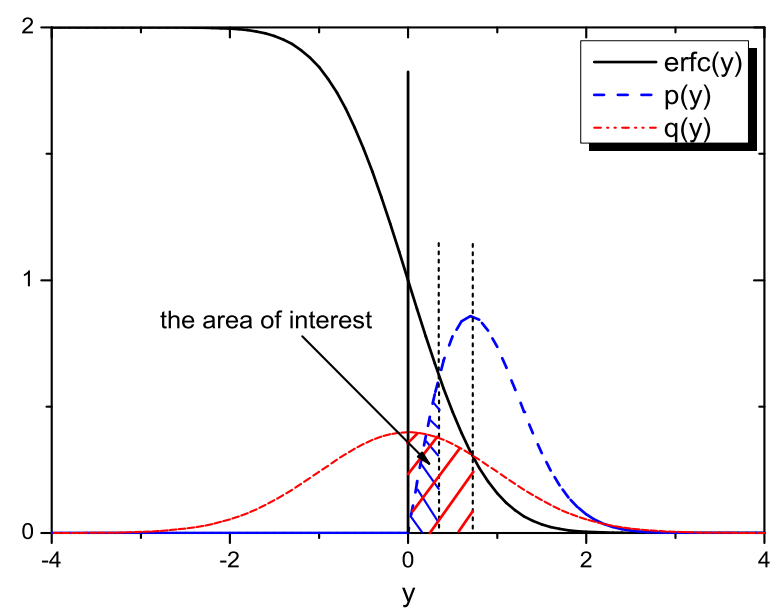

Fig. 3. An illustration for the choice of the proposed sampling distribution $q(y)$.

weight, the $B E R_{\mid \mathbf{X}_{j}, \mathbf{H}_{j}, \mathbf{w}_{j}}$ in (15) takes into account the probability of a channel occurrence by inserting the importance weights $w_{n}=p\left(y_{n}\right) / q\left(y_{n}\right)\left(y_{n}=\left|H_{n}\right| \geq 0\right)$ :

$$
\begin{aligned}
B E R_{\mid \mathbf{X}_{i}, \mathbf{H}_{i}, \mathbf{w}_{i}}=\frac{1}{2 N_{d}\left(m_{I}+m_{Q}\right) \sum_{n \in I_{d}} w_{n}} \cdot \\
\sum_{n, \beta, u} w_{n} \cdot\left(\sum_{y=1}^{F_{u, v}} \lambda_{u, v, y} \operatorname{erfc}\left(\Gamma_{u, v, y}\left(X_{n}, H_{n}\right)\right)\right. \\
\left.+\sum_{y=F_{u, v}+1}^{2^{u-1}} \rho_{u, v, y} \operatorname{erfc}\left(-\Gamma_{u, v, y}\left(X_{n}, H_{n}\right)\right)\right),
\end{aligned}
$$

where $p\left(y_{n}\right)$ is the probability density function of $\left|H_{n}\right|$, which is a Rayleigh distributed random variable with zero mean and variance $\sigma_{H}^{2}$ per dimension: $p\left(y_{n}\right)=y_{n} / \sigma_{H}^{2} \cdot e^{-y_{n}^{2} / 2 \sigma_{H}^{2}}$, and $q\left(y_{n}\right)$ is the sampling distribution. For this sampling distribution, we use a Gaussian distribution whose tail decays slower than the tail of $p\left(y_{n}\right): q\left(y_{n}\right)=1 / \sqrt{4 \pi \sigma_{H}^{2}} \cdot e^{-y_{n}^{2} / 4 \sigma_{H}^{2}}\left(y_{n} \geq\right.$ $0)$. This choice of $q\left(y_{n}\right)$ significantly reduces the number of runs required to evaluate the low BER values [15]. This can be explained as follows. Due to the shape of the Gaussian distribution, we generate with a high probability $\left|H_{n}\right|$ values with low amplitude, whereas the probability of obtaining this amplitude is low for the $p\left(y_{n}\right)$ distribution (see Fig. 3$)^{3}$. As a result, the error events, which will influence the accuracy most for low BERs values, are more likely to occur. This results in a smaller number of required simulation runs.

\section{NuMERICAL RESUlts}

In order to evaluate the accuracy of the proposed semianalytic BER evaluation procedures from (14) (SA-MC) and (15) (SA-IS), we compare the obtained numerical BER results with the empirical BER that is obtained by means of a BFMC simulation of the detector response to (3). This is done for various constellations, different $L$ and mismatch levels. Both AWGN and Rayleigh fading channels are considered. In the case of an AWGN channel, $H_{n}=1$ for all $n$. In the case of a Rayleigh fading channel, without loss of generality,

\footnotetext{
${ }^{3}$ Note that the negative values of $\left|H_{n}\right|$ yield $w_{n}=0$ and can therefore simply be omitted.
}

TABLE I. SIMULATION PARAMETERS

\begin{tabular}{|c|c|}
\hline Parameters & Reference values \\
\hline$E_{s}$ & 1 \\
\hline$N$ & 2048 \\
\hline$L$ & 2,4 or 8 \\
\hline$d g_{l}^{(100 \%)}$ & {$[0.61,-0.75,-0.31,0.26,0.82,-0.55,-0.16,-0.95]$} \\
\hline
\end{tabular}

we assume the Wide Sense Stationary Uncorrelated Scattering model and $2 \sigma_{H}^{2}=1$. Further, we generate $L$ independent gain mismatch values $d g_{l}^{(100 \%)}$ according to a uniform distribution over the interval $[-1,1]$ [8] and keep these values fixed. They can be interpreted as $100 \%$ level gain mismatch values corresponding to a particular TI-ADC implementation. The simulation parameters are summarized in Table I. The level of mismatch will be varied by scaling the $d g_{l}^{(100 \%)}$ gain mismatch values, i.e., for an $x \%$ mismatch level, we use as the gain mismatch values: $d g_{l}^{(x \%)}=\frac{x}{100} d g_{l}^{(100 \%)}$.

First, we investigate the BER performance in an AWGN channel $\left(H_{n}=1\right)$. In Fig. 4, BER curves obtained with the BF$\mathrm{MC}$ and SA-MC methods are provided for different mismatch levels, different modulation types and orders, and different number $L$ of sub-ADCs. For comparison, the BER for the same systems without mismatch (analytical results from [6]) and the approximate BER obtained by applying a Gaussian approximation (GA) [6] are also provided. Here, the number of simulation runs equals 100 for SA-MC and 1000 for BF$\mathrm{MC}$. These numbers of simulation runs are selected in order to have the estimated BER results converge. Further, the values $d g_{l}$ of the gain mismatch used in the simulations correspond to the first two $(L=2)$, four $(L=4)$ and eight $(L=8) d g_{l}$ values given in Table I, respectively. We make the following observations:

1) Mismatch versus no mismatch: It is obviously seen from Fig. 4 that the gain mismatch causes a system performance degradation, which is worse when either $L$, the mismatch level, or the modulation order increases.

2) $S A-M C$ versus $B F-M C$ and $G A$ : Fig. 4 illustrates that the proposed semi-analytic BER evaluation procedure SA-MC yields the same results as BF-MC, but with much less simulation runs, whereas the approximate BER from [6] significantly overestimates the actual BER for a low value $L$.

We investigated numerous other parameter settings (results not shown in this paper), and found the similar results.

Next, we demonstrate the efficiency of employing IS in evaluating low BER values in a Rayleigh fading channel ${ }^{4}$. Fig. 5 illustrates the BER resulting from BF-MC, SA-MC (obtained by using (14)) and SA-IS (obtained by using (15)) for 4-QAM, $0.5 \%$ mismatch and $L=4$. In order to have a convergence of the estimated BER results, the required number of simulation runs for the BF-MC, SA-MC and SA-IS approaches is 5000, 1000 and 100, respectively. By means of comparison, the BER obtained with SA-MC after only 100 simulation runs is also

${ }^{4}$ The channel impulse response used in the simulations is modelled as a conventional exponential decaying multi-path fading channel: $h_{k}=$ $C \sum_{m=0}^{99} e^{-m / 200} A_{m} \delta_{k-m}$, where $C$ is the normalization constant and $A_{m}$ are i.i.d complex-valued Gaussian random variables with zero mean and unit variance. The CP length equals 128 . 
(a)

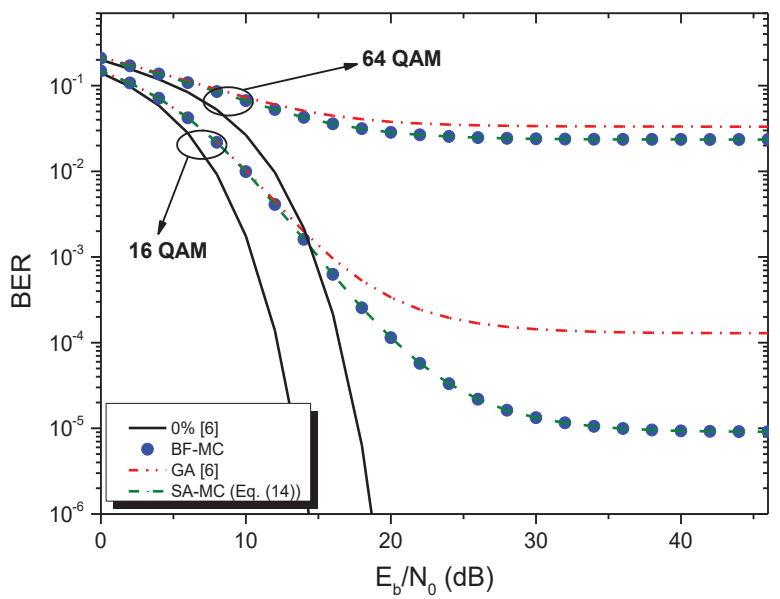

(b)

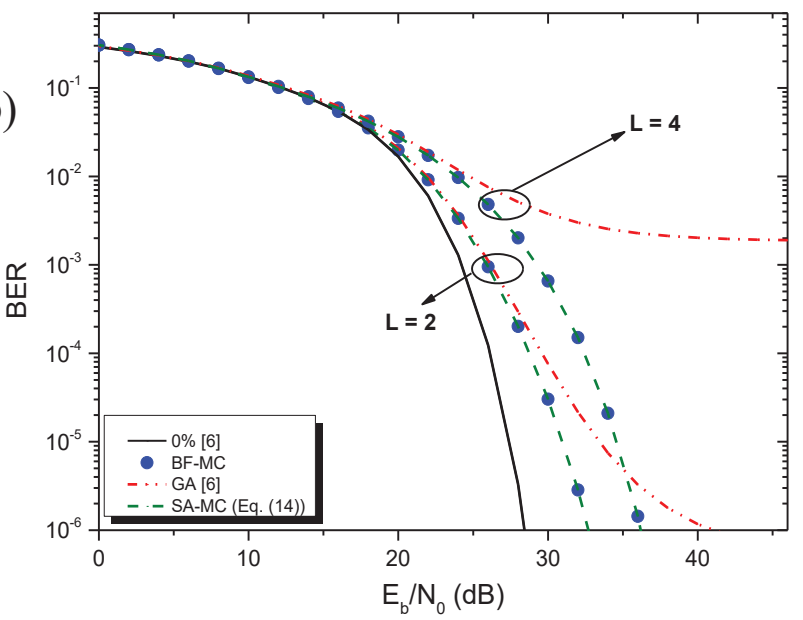

(c)

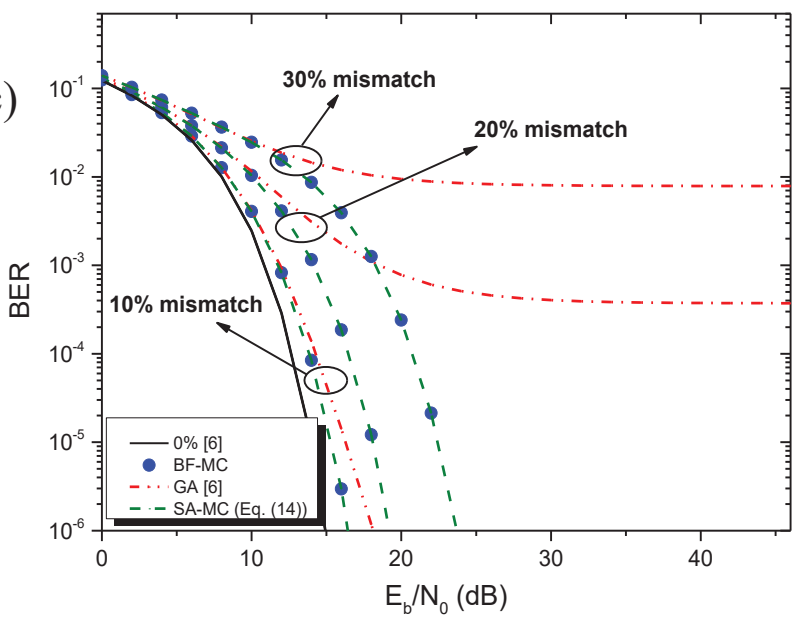

Fig. 4. BER curves in an AWGN channel for different mismatch levels, different modulation types and orders, and different $L$ : (a) square QAM, $20 \%$ mismatch, $L=8$; (b) 32 PAM, $3 \%$ mismatch, $L=2$, 4 ; (c) $4 \times 2$ QAM, $10 \%, 20 \%$ and $30 \%$ mismatch, $L=2$.

shown. It can be observed from Fig. 5 that the SA-MC BER with 100 runs significantly deviates from the BF-MC BER, while the SA-MC BER with 1000 runs and the SA-IS BER almost coincide with the BF-MC BER. Hence, SA-IS requires much less simulations to accurately estimate low BER, i.e., a factor 50 and 10 less as compared to BF-MC and SA-MC, respectively.

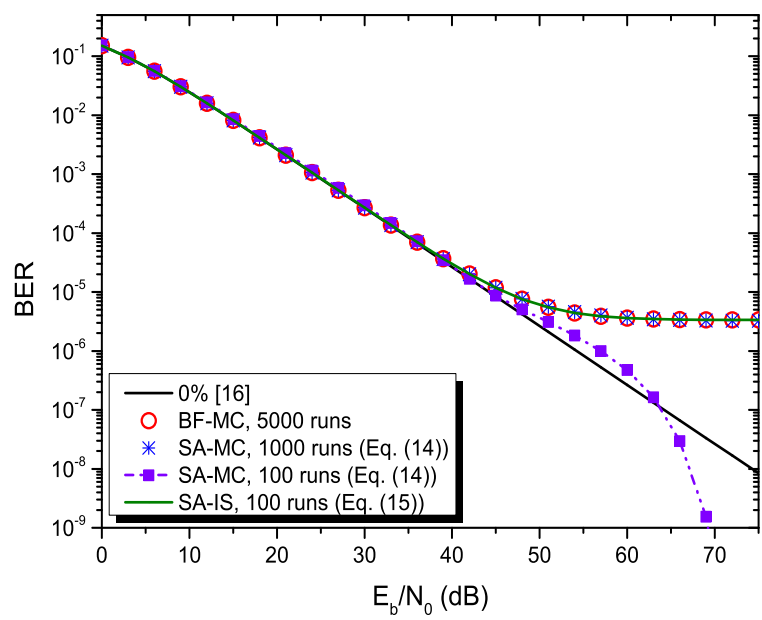

Fig. 5. BER curves in a Rayleigh fading channel for 4 QAM, $0.5 \%$ mismatch, $L=4$, and different estimators.

\section{COnclusions}

In this paper, we have proposed a novel semi-analytical procedure to efficiently evaluate the exact BER of OFDM systems with the effect of TI-ADC's gain mismatch for AWGN channels and Rayleigh fading channels. Simulation results confirmed the accuracy and efficiency of the proposed approach.

\section{ACKNOWLEDGMENT}

This research has been funded by the Interuniversity Attraction Poles Programme initiated by the Belgian Science Policy Office. The authors acknowledge the financial support from the Flemish fund for Scientific Research (FWO).

\section{REFERENCES}

[1] R. V. Nee and R. Prasad, OFDM for Wireless Multimedia Communications, Boston, MA: Artech House, 2000.

[2] W. C. Black and D. A. Hodges, "Time Interleaved Converter Arrays," IEEE J. Solid-State Circuits, vol. SSC-15, no. 6, pp. 1022-1029, Dec. 1980.

[3] A. Petraglia and S. K. Mitra, "Analysis of Mismatch Effects Among A/D Converters in a Time-Interleaved Waveform Digitizer," IEEE Trans. Inst. Meas. , pp. 831-835, Oct. 1991.

[4] N. Kurosawa, H. Kobayashi, K. Maruyama, H. Sugawara and K. Kobayashi, "Explicit Analysis of Channel Mismatch Effects in TimeInterleaved ADC Systems," IEEE Trans. Circuits Syst. , pp. 261-271, Mar. 2001.

[5] V.-T.-D. Huynh, N. Noels, P. Rombouts, J. Armstrong, and H. Steendam, "Effect of Time-Interleaved Analog-to-Digital Converter Mismatches on OFDM Performance," IEEE Inter. OFDM Workshop, pp. 128-135, Aug. 2014.

[6] V.-T.-D. Huynh, N. Noels and H. Steendam, "Approximate Bit Error Rate for High-Speed OFDM Systems Impaired by a Gain Mismatch of a TI-ADC Realization," 24 $4^{\text {th }}$ Inter. Conf. Telecomm., May 2017.

[7] H. Johansson and P. Lowenborg, "Reconstruction of Non-Uniformly Sampled Bandlimited Signals by Means of Digital fractional Delay Filters," IEEE Trans. Signal Proc., vol. 50, no. 11, pp. 2757-2767, Nov. 2002.

[8] S. Ponnuru, M. Seo, U. Madhow and M. Rodwell, "Joint Mismatch and Channel Compensation for High-Speed OFDM Receivers with TimeInterleaved ADCs," IEEE Trans. Comm., vol. 58, no. 8, pp. 2391-2401, Aug. 2010. 
[9] Y. S. Choi, P. J. Voltz and F. A. Cassara, "On Channel Estimation and Detection for Multicarrier Signals in Fast and Selective Rayleigh Fading Channels," IEEE Trans. Comm., vol. 6, no. 5, pp. 190-192, vol. 49, no. 8, pp. 1375-1376, Aug. 2001.

[10] I. Guvenc, S. Gezici, Z. Sahinoglu and U. C. Kozat, Reliable Communications for Short-range Wireless Systems, Cambridge University Press, USA, 2011.

[11] K. Cho, and D. Yoon, "On the General BER Expression of One- and Two-Dimensional Amplitude Modulations," IEEE Trans. Comm., vol. 50, no. 7, pp. 1074-1080, Jul. 2002.

[12] M. Gardner, Knotted Doughnuts and Other Mathematical Entertainments, W.H. Freeman \& Company, 1986 .

[13] P. K. Vitthaladevuni and M. S. Alouini, "A Closed-Form Expression for The Exact BER of Generalized PAM and QAM Constellations," IEEE Trans. Comm., vol. 52, no. 5, pp. 698-700, May 2004.

[14] D. Lu and K. Yao, "Improved Importance Sampling Technique for Efficient Simulation of Digital Communication Systems," IEEE J. Selected Area in Comm., vol. 6, no. 1, pp. 67-75, Jan. 1988.

[15] S. K. Ahn and K. Yang, "Importance Sampling for Performance Estimation of LDPC Codes over Rayleigh Fading Channels," $7^{\text {th }}$ Inter. Symp. Turbo Codes and Iterative Info. Proc., 2012

[16] K. D. Rao, Channel Coding Techniques for Wireless Communications, Springer, 2015. 